\title{
Correction: Validation of a preclinical model for assessment of drug efficacy in melanoma
}

\section{Julie Delyon, Mariana Varna, Jean-Paul Feugeas, Aurélie Sadoux, Saliha Yahiaoui, Marie-Pierre Podgorniak, Geoffroy Leclert, Sarra Mazouz Dorval, Nicolas Dumaz, Anne Janin, Samia Mourah and Céleste Lebbé}

Present: Due to an error during submission of the revised manuscript, Dr. Jean Soulier's name was omitted from the author list.

Corrected: Correct author list for this article is available below: Authors sincerely apologize for this oversight.

Original article: Oncotarget. 2016; 7(11): 13069-81. doi: 10.18632/oncotarget.7541.

\footnotetext{
Julie Delyon ${ }^{1,2,3}$, Mariana Varna ${ }^{4,5,6}$, Jean-Paul Feugeas ${ }^{3,7}$, Aurélie Sadoux ${ }^{1}$, Saliha Yahiaoui ${ }^{1}$, Marie-Pierre Podgorniak ${ }^{8}$, Geoffroy Leclert ${ }^{1}$, Sarra Mazouz Dorval ${ }^{3,9}$, Nicolas Dumaz ${ }^{1,3}$, Jean Soulier ${ }^{10,11}$, Anne Janin ${ }^{4,5,12}$, Samia Mourah ${ }^{1,3,8, *}$ and Céleste Lebbé ${ }^{1,2,3, *}$

${ }^{1}$ INSERM UMR_S976, Paris, F-75010, France

${ }^{2}$ AP-HP, Hôpital Saint-Louis, Department of Dermatology, Paris, F-75010, France

${ }^{3}$ Université Paris-Diderot, Sorbonne Paris Cité, Paris, F-75013, France

${ }^{4}$ INSERM UMR_S1165, Paris, F-75010, France

${ }^{5}$ Université Paris-Diderot, Department of Pathology, UMR_S1165, Paris, F-75010, France

${ }^{6}$ UMR CNRS 8612, Institut Galien-UFR de Pharmacie, Université de Paris-Sud, Châtenay-Malabry, F-92290, France

7 INSERM UMR_1137, Paris, F-75010, France

${ }^{8}$ AP-HP, Hôpital Saint-Louis, Laboratoire de Pharmacologie Biologique, Paris, F-75010, France

${ }^{9}$ AP-HP, Hôpital Saint-Louis, Department of Plastic, Reconstructive and Esthetic Surgery, Paris, F-75010, France

${ }^{10}$ AP-HP, Hôpital Saint-Louis, Department of Biological Hematology, Paris, F-75010, France

${ }^{11}$ Institute of Hematology (IUH), University Paris-Diderot, Paris, F-75010, France

${ }^{12}$ AP-HP, Hôpital Saint-Louis, Department of Pathology, Paris, F-75010, France

${ }^{*}$ These authors have contributed equally to this work
} 